\title{
1D Tube, 2D Layer and 3D Framework Derived from A New Series of Metal(II)-5-Aminodiacetic Isophthalate Coordination Polymers
}

Yanqing Xu, Daqiang Yuan, Benlai Wu, Mingyan Wu, Lei Han, Feilong Jiang, * and Maochun Hong

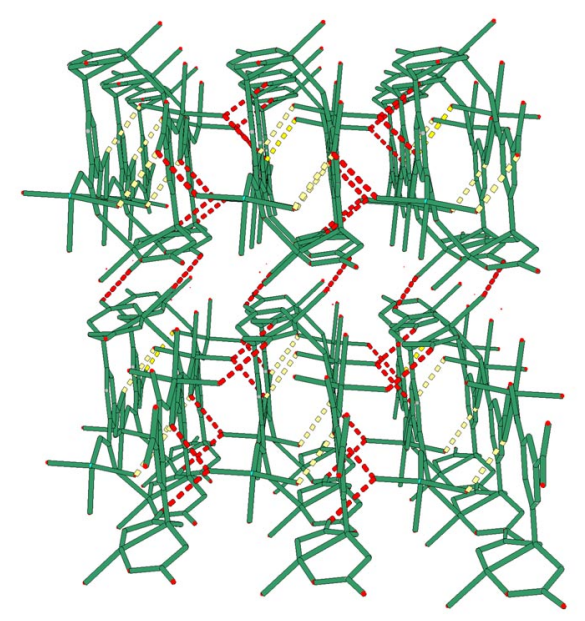

Figure S1. Packing of one-dimensional tubes with hydrogen bonds illustrated in 1. (red: intermolecular type, yellow: intramolecular type).

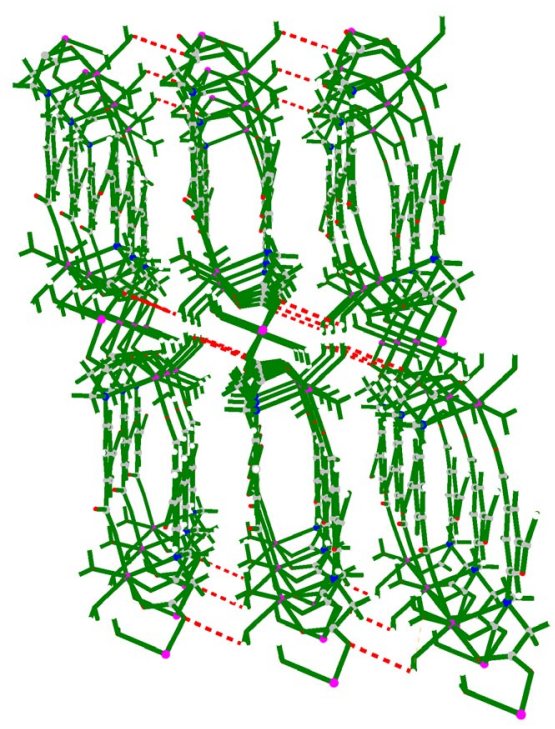

Figure S2. The 3-D supramolecular structure of $\mathbf{2}$ showing the linkage of intermolecular hydrogen bonds (red dashed line). 


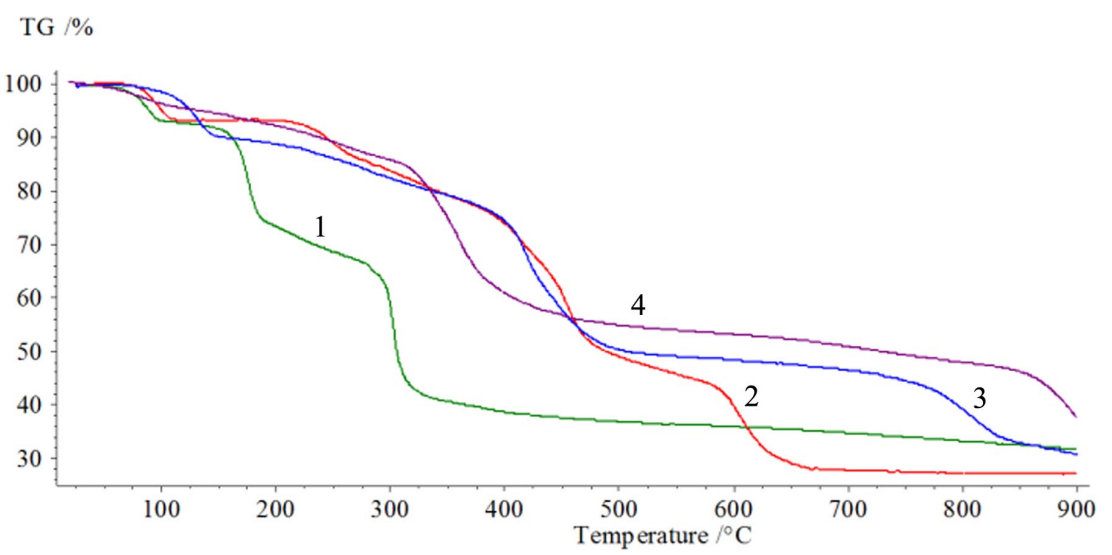

Figure S3. TGA curves. (green for $\mathbf{1}$, red for $\mathbf{2}$, blue for $\mathbf{3}$, purple (4) for free ligand) 